\title{
Dynamic Seeding and in Vitro Culture of Hepatocytes in a Flow Perfusion System
}

\author{
STEPHEN S. KIM, M.D., ${ }^{1,2}$ CATHRYN A. SUNDBACK, Sc.D., ${ }^{1}$ \\ SATOSHI KAIHARA, M.D., ${ }^{1}$ MARK S. BENVENUTO, B.S., ${ }^{1}$ BYUNG-SOO KIM, M.S., ${ }^{3}$ \\ DAVID J. MOONEY, Ph.D., ${ }^{3}$ and JOSEPH P. VACANTI, M.D. ${ }^{1}$
}

\begin{abstract}
Our laboratory has investigated hepatocyte transplantation using biodegradable polymer matrices as an alternative treatment to end-stage liver disease. One of the major limitations has been the insufficient survival of an adequate mass of transplanted cells. This study investigates a novel method of dynamic seeding and culture of hepatocytes in a flow perfusion system. In experiment I, hepatocytes were flow-seeded onto PGA scaffolds and cultured in a flow perfusion system for $24 \mathrm{~h}$. Overall metabolic activity and distribution of cells were assessed by their ability to reduce MTT. DNA quantification was used to determine the number of cells attached. Culture medium was analyzed for albumin content. In Experiment II, hepatocyte/polymer constructs were cultured in a perfusion system for 2 and 7 days. The constructs were examined by SEM and histology. Culture medium was analyzed for albumin. In experiment I, an average of $4.4 \times 10^{6}$ cells attached to the scaffolds by DNA quantification. Cells maintained a high metabolic activity and secreted albumin at a rate of 13 $\mathrm{pg} / \mathrm{cell} / \mathrm{day}$. In experiment II, SEM demonstrated successful attachment of hepatocytes on the scaffolds after 2 and 7 days. Cells appeared healthy on histology and maintained a high rate of albumin secretion through day 7 . Hepatocytes can be dynamically seeded onto biodegradable polymers and survive with a high rate of albumin synthesis in the flow perfusion culture system.
\end{abstract}

\section{INTRODUCTION}

$\mathbf{E}$ ACH Year 26,000 people die of end-stage liver disease (ESLD) in the United States with an estimated annual cost of $\$ 9$ billion. ${ }^{1}$ Liver transplantation is currently the only established successful treatment for ESLD. The severe scarcity of donor organs, especially in the pediatric population, has become a major limitation and has stimulated investigation into selective cell transplantation. ${ }^{2,3}$ Using the principles of tissue engineering, our laboratory has investigated hepatocyte transplantation using three dimensional

\footnotetext{
${ }^{1}$ Laboratory for Transplantation and Tissue Engineering, Children's Hospital and Harvard Medical School, Boston, Massachusetts.

${ }^{2}$ Department of Surgery, University of Chicago Hospitals, Chicago, Illinois.

${ }^{3}$ Department of Chemical Engineering, University of Michigan, Ann Arbor, Michigan.
} 
biodegradable polymer matrices (3DP) as a novel approach to the treatment of ESLD. ${ }^{4,5}$ To be successful, several achievements must be made, including: (a) sufficient mass of transplanted cells must become engrafted, (b) hepatocyte survival must be maintained, (c) transplanted cells must proliferate, (d) transplanted cells must remain functional, and (e) ongoing hepatotrophic stimulation from the portal circulation must be provided.

Previous studies performed in this laboratory have demonstrated survival of hepatocytes transplanted on biodegradable polymer discs in peripheral tissue sites, ${ }^{6,7}$ improvement in engraftment and survival of transplanted hepatocytes with hepatotrophic stimulation using portacaval shunt and partial hepatectomy, ${ }^{8-10}$ partial correction of single enzyme liver defects, ${ }^{11}$ and total correction of hyperbilirubinemia in the rat model with $10-15 \%$ of the normal liver mass. ${ }^{12}$ One of the major limitations has been the insufficient survival of an adequate mass of transplanted cells to permanently correct defects in liver function. We hypothesized that the limitations of oxygen/nutrient diffusion and waste exchange are critical factors for the highly metabolically active cells during the initial posttransplantation period. To address this issue, we have designed and fabricated a complex 3DP with an intrinsic network of interconnected channels. ${ }^{13,14}$ Preliminary studies have demonstrated successful attachment of hepatocytes on the complex polymer scaffold in large numbers and survival with a high rate of albumin production under flow conditions compared to static conditions in vitro. ${ }^{14}$ Recently, significant improvements in cell attachment and function have been demonstrated using dynamic seeding techniques for a variety of cells. ${ }^{15-17}$ The purpose of the current study was to investigate a novel method of dynamic hepatocyte seeding onto 3DP and to examine the survival and function of hepatocytes in a flow perfusion culture system.

\section{MATERIALS AND METHODS}

\section{Animals}

In-bred, male, Lewis rats (Charles River Laboratories, Wilmington, MA) weighing 150-300 g were used in all experiments. Animals were housed in the Animal Research Facility of Children's Hospital, Boston, Massachusetts, in accordance with NIH guidelines for the care of laboratory animals. Rats were maintained in a temperature-regulated environment on a 12-h light/dark cycle and given access to rat chow and tap water ad libitum.

\section{Polymer Fabrication}

Microporous three-dimensional synthetic biodegradable polymer tubes were fabricated from nonwoven sheets of polyglycolic acid (PGA) fibers (fiber diameter, $12 \mu \mathrm{m}$; mesh thickness, $2 \mathrm{~mm}$; bulk density, 60 $\mathrm{mg} / \mathrm{cm}^{3}$; porosity, 96\%; mean pore size, $250 \mu \mathrm{m}$; Smith and Nephew, Heslington, York, U.K.) sprayed on the outer surfaces with a 5\% solution of polylactide-co-glycolide (PLGA, 85:15; Medisorb, Cincinnati, OH) as previously described. ${ }^{18}$

\section{Flow Perfusion Culture System}

A flow perfusion culture system created in our laboratory was modified using three-way stopcocks to allow the addition of dynamic cell-seeding loops in-line with the culture loops. The culture medium was pumped at a flow rate of $1.5 \mathrm{~mL} / \mathrm{min}$ from a $100-\mathrm{mL}$ reservoir, through the oxygenation tubing and the cell/polymer construct housing unit, and recirculated back to the reservoir. The flow rate needed for cell survival was estimated based on cell mass and reported values for nutrient consumption. The entire seeding/perfusion unit was sterilized by autoclave and maintained at $37^{\circ} \mathrm{C}$ with $5 \% \mathrm{CO}_{2}$ supplementation.

\section{Cell Isolation}

Adult, male Lewis rats were used as cell donors. Hepatocytes (HC) were isolated using a modification of the two-step collagenase perfusion procedure as previously described. ${ }^{19}$ Cell counts and viability were determined using trypan blue exclusion test. 


\section{Flow Seeding/Flow Perfusion Culture}

Isolated HC were resuspended in warmed chemically defined hepatocyte growth medium (HGM) supplemented with epidermal growth factor (EGF; Collaborative Biomedical Products, Bedford, MA) as previously described ${ }^{20}$ at a density of $5 \times 10^{6}$ cells $/ \mathrm{mL}$. Twenty-five milliliters of the cell suspension was placed in the reservoir bottle, and the $\mathrm{HC}$ were suspended in culture medium using a magnetic stir bar and stirrer at $110 \mathrm{rpm}$. HC were flow-seeded onto the 3DP placed within the construct housing unit at a flow rate of $1.5 \mathrm{~mL} / \mathrm{min}$ for $4 \mathrm{~h}$. After the flow-seeding period, the reservoir bottle containing the cell suspension was replaced with fresh warmed culture medium and the loops flushed for $30 \mathrm{~min}$ to remove any excess cells. After the flush period, the reservoir bottles were replaced again with fresh warmed HGM for flow perfusion culture.

\section{Study Design}

Experiment I. HC were flow-seeded onto 3DP $(n=12)$ for $4 \mathrm{~h}$. After the seeding and flushing period, HC were cultured under flow conditions for $24 \mathrm{~h}$ in $50 \mathrm{~mL}$ of fresh warmed culture medium. After $24 \mathrm{~h}$, the culture medium was analyzed for albumin by enzyme-linked immunosorbent assay (ELISA) as previously described, ${ }^{14}$ and the reservoir bottle was replaced with fresh warmed culture medium containing 1\% 3-[4,5-dimethylthiazol-2-yl]-2,5-diphenyltetrazolium bromide (MTT: Sigma Chemical Co., St. Louis, MO) to assess general metabolic activity and distribution of HC. HC were incubated in MTT solution under flow conditions for an additional $12 \mathrm{~h}$. All constructs were harvested after the MTT incubation period and homogenized. DNA content was measured by the diphenylamine method as previously described. ${ }^{21,22} \mathrm{HC}$ suspensions of varying cell concentration were used as standards to derive the number of cells attached to the 3DP.

Experiment II. HC were flow-seeded onto 3DP for $4 \mathrm{~h}$. After the seeding and flushing period, HC were cultured under flow conditions for 2 days (group A, $n=8$ ) and 7 days (group B, $n=4$ ). Constructs in group A were harvested after 2 days and processed for scanning electron microscopy (SEM) and histology as previously described. ${ }^{14}$ Culture medium was analyzed for albumin by ELISA. Constructs in group B were harvested after 7 days of perfusion culture and processed for SEM and histology. Culture medium in Group B was replaced after the first $24 \mathrm{~h}$, then every $48 \mathrm{~h}$ thereafter. Culture medium was sampled at days 3,5 , and 7 for albumin content.

\section{RESULTS}

\section{Polymer Scaffold Fabrication}

The 3DP were fabricated in the shape of a tube with dimensions of 5-mm outer diameter, 2-mm inner diameter, and 5-mm length, with a microporosity of $96 \%$ and average micropore diameter of $250 \mu \mathrm{m}$. The outer surface coating of PLGA provided structural integrity to the polymer tube.

\section{Flow Perfusion Culture System}

The flow rate needed for cell survival was estimated based on reported values for nutrient consumption and cell mass. Under standard culture conditions, oxygen becomes the limiting nutrient. The volumetric oxygen consumption rate $\left(\mathrm{Q}_{\mathrm{O} 2}\right)$ is approximately $2-6 \times 10^{-5} \mathrm{mmol} / \mathrm{cm}^{3}$ cell mass-second for metabolically active cells. ${ }^{23}$ The 3DP contains $<30 \%$ by volume cells after cell seeding, and the scaffold void volume $(\mathrm{V})$ is $0.079 \mathrm{~cm}^{3}$. We specified that the oxygen concentration should not decrease more than $50 \%$ from the inflow to the outflow of the cell/polymer housing unit. The flow rate $(F)$ needed to maintain the specified oxygen concentration after cell seeding is thus estimated by: $\mathrm{F}=(0.3)(\mathrm{V})\left(\mathrm{Q}_{\mathrm{O} 2}\right) /(0.5)\left(\mathrm{C}_{\mathrm{O} 2}\right.$ inflow $)$, for a given value of inflow oxygen concentration $\left(\mathrm{C}_{\mathrm{O} 2}\right.$ inflow $)$. The oxygen concentration in the $5 \% \mathrm{CO}_{2} /$ humidified airsaturated culture medium at $37^{\circ} \mathrm{C}$ is $1.6 \times 10^{4} \mathrm{mmole} / \mathrm{mL}$. Therefore, the flow rate needed for survival of the initial cell mass is estimated to be at most $1 \mathrm{~mL} / \mathrm{min}$. The flow rate in our perfusion system was set at $1.5 \mathrm{~mL} / \mathrm{min}$ to ensure adequate oxygen delivery. 


\section{Cell Isolation}

All cell isolations yielded $4-7 \times 10^{8} \mathrm{HC}$ per isolation with greater than $85 \%$ viability. The viability of $\mathrm{HC}$ in the culture medium after 4 hours of dynamic seeding remained greater than $75 \%$.

\section{Specimen Analysis}

Experiment I. The general metabolic activity and distribution of $\mathrm{HC}$ were assessed by the ability of live cells to reduce MTT. HC maintained high metabolic activity in all of the cell/polymer constructs as demonstrated by prominent staining after incubation in MTT. Live HC were uniformly distributed throughout the 3DP on gross examination. After $4 \mathrm{~h}$ of dynamic seeding and $24 \mathrm{~h}$ of flow perfusion culture, the mean number of HC attached to the 3DP was calculated to be approximately $4.43 \pm 1.3 \times 10^{6}$ cells by DNA quantification. Albumin secretion was calculated to be approximately $13 \pm 4 \mathrm{pg} /$ cell by ELISA.

Experiment II. SEM demonstrated successful attachment of large numbers of HC on the surfaces of the PGA fibers within the 3DP after 2 and 7 days in flow conditions (Fig. 1). SEM confirmed the uniform distribution of HC on the inner surfaces, outer surfaces, and through the bulk of the polymer scaffold. Histology demonstrated viable $\mathrm{HC}$ distributed throughout the polymer scaffold, maintaining both cell-cell and cell-matrix interactions after 2 and 7 days. Based on the calculated number of hepatocytes attached to the 3DP $\left(4.43 \times 10^{6}\right.$ cells $)$ derived from experiment I, albumin secretion was calculated to be approximately $9 \pm 3 \mathrm{pg} / \mathrm{cell} / \mathrm{day}$ at day 2 for constructs in group A; and $6.2 \pm 3 \mathrm{pg} /$ cell/day at day $3 ; 13.1 \pm 12 \mathrm{pg} / \mathrm{cell} / \mathrm{day}$ at day 5 ; and $17.2 \pm 13 \mathrm{pg} / \mathrm{cell} / \mathrm{day}$ at day 7 for constructs in group $\mathrm{B}$.

\section{DISCUSSION}

Our laboratory has been investigating heterotopic hepatocyte transplantation using three-dimensional synthetic biodegradable polymer matrices as a novel approach to the treatment of end-stage liver disease. One of the major limitations has been the insufficient survival of a large number of transplanted hepatocytes to permanently replace liver function. We have hypothesized that the limiting factor may be the inadequate

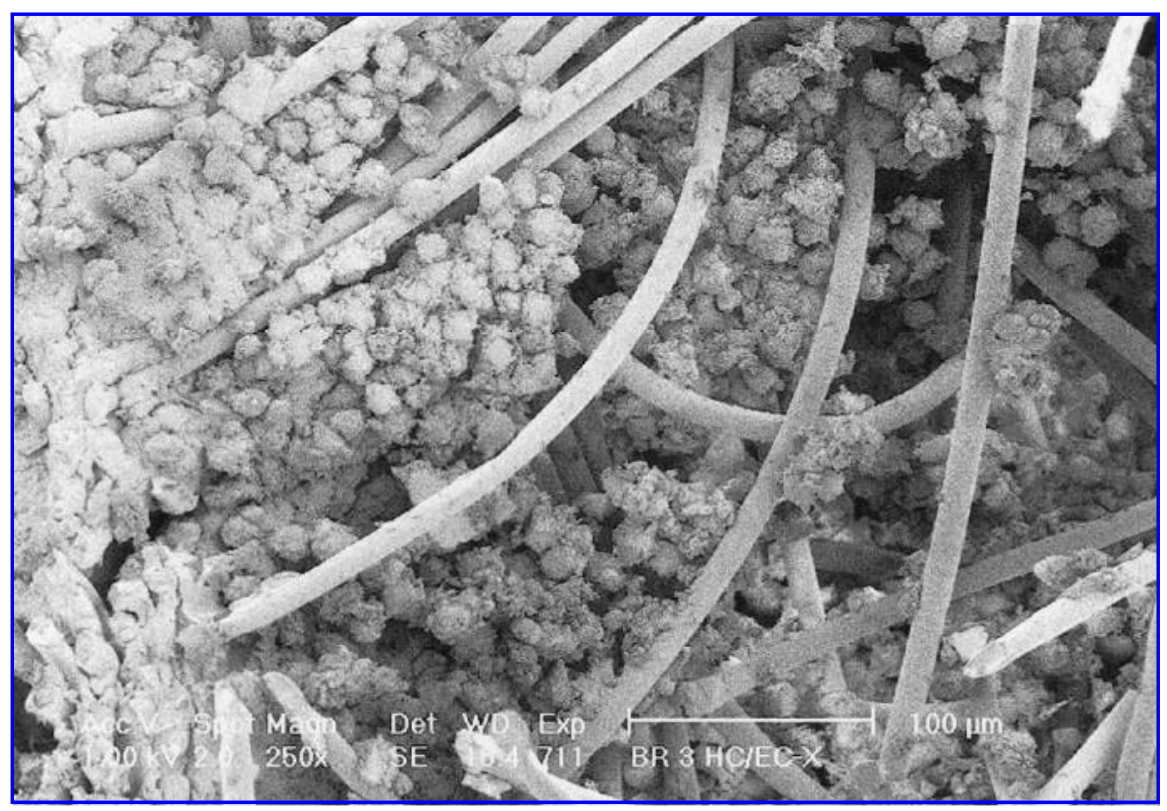

FIG. 1. SEM of hepatocytes attached to the PGA polymer matrix after 2 days. Original magnification, $\times 250$. 
diffusion of oxygen and nutrients to the cells on the polymer matrix initially after implantation until adequate neovascularization has occurred. To address this issue, we have fabricated complex three-dimensional biodegradable polymer scaffolds with a high surface area-to-volume ratio and microporosity. In this study, we have investigated a novel method of dynamic hepatocyte seeding and flow perfusion culture, and demonstrated the attachment, survival, and function of hepatocytes on the complex polymer scaffolds under flow conditions.

Previous studies performed in this laboratory have demonstrated survival of hepatocytes transplanted on thin polymer discs in peripheral tissue sites such as the omentum, mesentery, and subcutaneous tissue, and the partial correction of single enzyme liver defects. The main obstacle has been the survival of only a small number of the transplanted cells. Recently, we have fabricated highly porous three-dimensional polymer scaffolds and demonstrated improved hepatocyte function on these matrices when cultured under flow conditions. The larger size and the high porosity of these devices contribute a much larger surface area for cell attachments compared to the previously studied polymer discs. The three-dimensional design provides a structural template to guide cellular organization, enhance neovascularization, and increase the capacity for oxygen/nutrient delivery. Conditioning the cells in a flow perfusion system in vitro may provide important cues for cell-cell and cell-matrix interaction and remodeling.

Optimization of hepatocyte seeding onto these polymer devices is critical for maximizing the mass of cells that can be transplanted and for uniformly distributing the cells throughout the polymer scaffold. Many investigators have demonstrated the beneficial effects of dynamic seeding techniques in regards to cell attachment, uniform cell distribution within the polymer, and long-term survival and function in a variety of cell types. In the current study, we have designed a simple system for dynamic hepatocyte seeding, demonstrated successful attachment and uniform distribution throughout the polymer scaffold, and survival of a large number of cells after 7 days of flow culture. In addition, the hepatocytes demonstrated an increasing rate of albumin synthesis over the culture period from $6.2 \mathrm{pg} / \mathrm{cell} /$ day to $17.2 \mathrm{pg} / \mathrm{cell} / \mathrm{day}$. The rate of albumin secretion in the normal rat liver in-vivo has been estimated to be approximately 140-170 $\mathrm{pg} / \mathrm{cell} / \mathrm{day} .{ }^{24,25}$ Although our values of albumin secretion are significantly lower than in vivo liver rates, they are comparable to rates reported in other studies investigating long-term hepatocyte culture and extracorporeal liver assist devices. ${ }^{26-30}$ More importantly, however, while most studies report a trend of declining rates of albumin secretion over time, the current study has demonstrated a nearly threefold increase in albumin secretion rate over the culture period. Continued investigation into the optimization of the polymer scaffold, the dynamic hepatocyte seeding parameters, and long-term in vitro culture under flow conditions may yield many insights into the mechanisms involved in tissue morphogenesis and regeneration, and may ultimately lead to the development of a permanent implantable device to replace liver function.

\section{ACKNOWLEDGMENTS}

This work was funded by generous grants from Advanced Tissue Sciences, the Holly Ann Soulard Research Fund, and the Thomas Anthony Pappas Charitable Foundation. The article was presented at the Second Bi-Annual Meeting of the Tissue Engineering Society, December 4-6, 1998, Orlando, Florida.

\section{REFERENCES}

1. American Liver Foundation. Fact Sheet: Hepatitis, Liver, and Gallbladder Diseases in the United States. Cedar Grove, NJ: American Liver Foundation, 1997.

2. Russell, P.S. Selective transplantation. Ann. Surg. 201, 255, 1985.

3. Vacanti, J.P. Beyond transplantation. Arch. Surg. 123, 545, 1988.

4. Vacanti, J.P., Morse, M.A., Saltzman, W.M., Domb, A.J., Perez-Atayde, A., and Langer, R. Selective cell transplantation using bioabsorbable artificial polymers as matrices. J. Pediatr. Surg. 23, 3, 1988.

5. Langer, R., and Vacanti, J.P. Tissue engineering. Science 260, 920, 1993.

6. Mooney, D.J., Kaufmann, P.M., Sano, K., McNamara, K., Vacanti, J.P., and Langer, R. Transplantation of hepatocytes using porous, biodegradable sponges. Transplant. Proc. 26, 3425, 1994. 


\section{KIM ET AL.}

7. Mooney, D.J., Park, S., Kaufmann, P.M., et al. Biodegradable sponges for hepatocyte transplantation. J. Biomed. Mater. Res. 29, 959, 1995.

8. Sano, K., Cusick, R.A., Lee, H., et al. Regenerative signals for heterotopic hepatocyte transplantation. Transplant. Proc. 28, 1859, 1996.

9. Kaufmann, P.M., Sano, K., Uyama, S., Schloo, B., and Vacanti, J.P. Heterotopic hepatocyte transplantation using three-dimensional polymers: evaluation of the stimulatory effects by portacaval shunt or islet cell cotransplantation. Transplant. Proc. 26, 3343, 1994.

10. Kaufmann, P.M., Sano, K., Uyama, S., Takeda, T., and Vacanti, J.P. Heterotopic hepatocyte transplantation: assessing the impact of hepatotrophic stimulation. Transplant. Proc. 26, 2240, 1994.

11. Takeda, T., Kim, T.H., Lee, S.K., Langer, R., and Vacanti, J.P. Hepatocyte transplantation in biodegradable polymer scaffolds using the dalmatian dog model of hyperuricosuria. Transplant. Proc. 27, 635, 1995.

12. Asonuma, K., Gilbert, J.C., Stein, J.E., Takeda, T., and Vacanti, J.P. Quantitation of transplanted hepatic mass necessary to cure the Gunn rat model of hyperbilirubinem ia. J. Pediatr. Surg. 27, 298, 1992.

13. Griffith, L.G., Wu, B.M., Cima, M.J., Chaignaud, B., and Vacanti, J.P. In vitro organogenesis of liver tissue. Ann. N.Y. Acad. Sci. 831, 382, 1997.

14. Kim, S.S., Utsunomiya, H., Koski, J.A., et al. Survival and function of hepatocytes on a novel three-dimension al synthetic biodegradable polymer scaffold with an intrinsic network of channels. Ann. Surg. 228, $8,1998$.

15. Vunjak-Novak ovic, G., Freed, L.E., Biron, R.J., and Langer, R. Effects of mixing on the composition and morphology of tissue-engineered cartilage. A.I.Ch.E. J. 42, 850, 1996.

16. Vunjak-Novak ovic, G., Obradovic, B., Martin, I., Bursac, P.M., Langer, R., and Freed, L.E. Dynamic cell seeding of polymer scaffolds for cartilage tissue engineering. Biotechnol. Prog. 14, 193, 1998.

17. Kim, B.S., Putnam, A.J., Kulik, T.J., and Mooney, D.J. Optimizing seeding and culture methods to engineer smooth muscle tissue on biodegradable polymer matrices. Biotechnol. Bioeng. 57, 46, 1998.

18. Mooney, D.J., Mazzoni, C.L., Breuer, C., et al. Stabilized polyglycolic acid fiber-based tubes for tissue engineering. Biomaterials 17, 115, 1996.

19. Aiken, J., Cima, L., Schloo, B., et al. Studies in rat liver perfusion for optimal harvest of hepatocytes. $\underline{\text { J. Pediatr. }}$ Surg. 25, 140, 1990.

20. Block, G.D., Locker, J., Bowen, W.C., et al. Population expansion, clonal growth, and specific differentiation patterns in primary cultures of hepatocytes induced by HGF/SF, EGF and TGF $\alpha$ in a chemically defined (HGM) medium. J. Cell Biol. 132, 1133, 1996.

21. Ceriotti, G. Determination of nucleic acids in animal tissues. J. Biol. Chem. 214, 59, 1955.

22. Giles, K.W., and Myers, A. An improved diphenylamine method for the estimation of deoxyribonucleic acid. Nature 206, 93, 1965.

23. Cima, L.G., Wilke, C.R., and Blanch, H.W. A theoretical and experimental evaluation of a novel radial-flow hollow fiber reactor for mammalian cell culture. Bioprocess Eng. 5, 19, 1990.

24. Cima, L.G., Langer, R., and Vacanti, J.P. Polymers for tissue and organ culture. J. Bioactive Compatible Polymers 6, 232, 1991.

25. Mooney, D., Hansen, L., Vacanti, J.P., Langer, R., Farmer, S., and Ingber, D. Switching from differentiation to growth in hepatocytes: control by extracellular matrix. J. Cell Physiol. 151, 497, 1992.

26. Kaufmann, P.M., Heimrath, S., Kim, B.S., and Mooney, D.J. Highly porous polymer matrices as a three-dimensional culture system for hepatocytes. Cell Transplant. 6, 463, 1997.

27. Shatford, R.A., Nyberg, S.L., Meier, S.J., et al. Hepatocyte function in a hollow fiber bioreactor: A potential bioartificial liver. J. Surg. Res. 53, 549, 1992.

28. Bader, A., Knop, E., Fruharf, N., et al. Reconstruction of liver tissue in vitro: geometry of characteristic flat bed, hollow fiber, and spouted bed bioreactors with reference to the in vivo liver. Artif. Organs 19, 941, 1995.

29. Wu, J.F., Friend, J.R., Hsiao, C.C., et al. Efficient assembly of rat hepatocyte spheroids for tissue engineering applications. Biotech. Bioeng. 50, 404, 1996.

30. Nyberg, S.L., and Misra, S.P. Hepatocyte liver-assist systems-a clinical update. Mayo Clin. Proc. 73, $765,1998$.

Address reprint requests to: Joseph P. Vacanti, M.D. Department of Surgery Massachusetts General Hospital 100 Fruit St. Boston, MA 02114

E-mail: jvacanti@partners.org 


\section{This article has been cited by:}

1. Richard W. Forsey, Julian B. Chaudhuri. 2009. Validity of DNA analysis to determine cell numbers in tissue engineering scaffolds. Biotechnology Letters 31:6, 819-823. [CrossRef]

2. Irena Shvartsman , Tal Dvir , Tamar Harel-Adar , Smadar Cohen . 2009. Perfusion Cell Seeding and Cultivation Induce the Assembly of Thick and Functional Hepatocellular Tissue-like ConstructPerfusion Cell Seeding and Cultivation Induce the Assembly of Thick and Functional Hepatocellular Tissue-like Construct. Tissue Engineering Part A 15:4, 751-760. [Abstract] [PDF] [PDF Plus]

3. Benjamin J. Lawrence, Mamatha Devarapalli, Sundararajan V. Madihally. 2009. Flow dynamics in bioreactors containing tissue engineering scaffolds. Biotechnology and Bioengineering 102:3, 935-947. [CrossRef]

4. Tammy T. Chang, Millie Hughes-Fulford . 2009. Monolayer and Spheroid Culture of Human Liver Hepatocellular Carcinoma Cell Line Cells Demonstrate Distinct Global Gene Expression Patterns and Functional PhenotypesMonolayer and Spheroid Culture of Human Liver Hepatocellular Carcinoma Cell Line Cells Demonstrate Distinct Global Gene Expression Patterns and Functional Phenotypes. Tissue Engineering Part A 15:3, 559-567. [Abstract] [Full Text] [PDF] [PDF Plus]

5. F. Wen, S. Chang, Y. C. Toh, T. Arooz, L. Zhuo, S. H. Teoh, H. Yu. 2008. Development of dual-compartment perfusion bioreactor for serial coculture of hepatocytes and stellate cells in poly(lactic- co -glycolic acid)-collagen scaffolds. Journal of Biomedical Materials Research Part B: Applied Biomaterials 87B:1, 154-162. [CrossRef]

6. Dajiang Du, Katsuko Furukawa, Takashi Ushida. 2008. Oscillatory perfusion seeding and culturing of osteoblast-like cells on porous beta-tricalcium phosphate scaffolds. Journal of Biomedical Materials Research Part A 86A:3, 796-803. [CrossRef]

7. Norihiro Matsumoto, Hitomi Hiruma, Shoji Nagaoka, Koutarou Fujiyama, Arata Kaneko, Hiroyoshi Kawakami. 2008. Cell processing on polyimide surface patterned by rubbing. Polymers for Advanced Technologies 19:8, 1002-1008. [CrossRef]

8. Jason D. Roh , Gregory N. Nelson, Brooks V. Udelsman , Matthew P. Brennan, Britt Lockhart , Peter M. Fong, Reynold I. Lopez-Soler, W. Mark Saltzman , Christopher K. Breuer . 2007. Centrifugal Seeding Increases Seeding Efficiency and Cellular Distribution of Bone Marrow Stromal Cells in Porous Biodegradable ScaffoldsCentrifugal Seeding Increases Seeding Efficiency and Cellular Distribution of Bone Marrow Stromal Cells in Porous Biodegradable Scaffolds. Tissue Engineering 13:11, 2743-2749. [Abstract] [PDF] [PDF Plus]

9. Nicholas E. Timmins , Arnaud Scherberich , Jennifer-Annika Früh , Michael Heberer , Ivan Martin , Marcel Jakob . 2007. Three-Dimensional Cell Culture and Tissue Engineering in a T-CUP (Tissue Culture Under Perfusion)Three-Dimensional Cell Culture and Tissue Engineering in a T-CUP (Tissue Culture Under Perfusion). Tissue Engineering 13:8, 2021-2028. [Abstract] [PDF] [PDF Plus]

10. Jose F. Alvarez-Barreto, Shawna M. Linehan, Robert L. Shambaugh, Vassilios I. Sikavitsas. 2007. Flow Perfusion Improves Seeding of Tissue Engineering Scaffolds with Different Architectures. Annals of Biomedical Engineering 35:3, 429-442. [CrossRef]

11. Anli Ouyang, Shang-Tian Yang. 2007. Effects of mixing intensity on cell seeding and proliferation in three-dimensional fibrous matrices. Biotechnology and Bioengineering 96:2, 371-380. [CrossRef] 
12. Rebecca A Penkala, Stephen S Kim. 2007. Gastrointestinal tissue engineering. Expert Review of Medical Devices 5:1, 65-72. [CrossRef]

13. Prakash Chandrasekaran, Chris Seagle, Lisa Rice, Jeff Macdonald, David A. Gerber . 2006. Functional Analysis of Encapsulated Hepatic Progenitor CellsFunctional Analysis of Encapsulated Hepatic Progenitor Cells. Tissue Engineering 12:7, 2001-2008. [Abstract] [PDF] [PDF Plus]

14. Luis A. Solchaga , Enrico Tognana , Kitsie Penick, Harihara Baskaran , Victor M. Goldberg , Arnold I. Caplan , Jean F. Welter . 2006. A Rapid Seeding Technique for the Assembly of Large Cell/Scaffold Composite ConstructsA Rapid Seeding Technique for the Assembly of Large Cell/Scaffold Composite Constructs. Tissue Engineering 12:7, 1851-1863. [Abstract] [PDF] [PDF Plus]

15. Prakash Chandrasekaran, Chris Seagle, Lisa Rice, Jeff MacDonald, David A. Gerber. 2006. Functional Analysis of Encapsulated Hepatic Progenitor Cells. Tissue Engineering, ahead of print060802052515020. [CrossRef]

16. Luis A. Solchaga, Enrico Tognana, Kitsie Penick, Harihara Baskaran, Victor M. Goldberg, Arnold I. Caplan, Jean F. Welter. 2006. A Rapid Seeding Technique for the Assembly of Large Cell/Scaffold Composite Constructs. Tissue Engineering, ahead of print060802052515026. [CrossRef]

17. Xiaohong Wang, Yongnian Yan, Yuqiong Pan, Zhuo Xiong, Haixia Liu, Jie Cheng, Feng Liu, Feng Lin, Rendong Wu, Renji Zhang, Qingping Lu. 2006. Generation of Three-Dimensional Hepatocyte/Gelatin Structures with Rapid Prototyping System. Tissue Engineering, ahead of print060127071904002. [CrossRef]

18. Xiaohong Wang, Yongnian Yan, Yuqiong Pan, Zhuo Xiong, Haixia Liu, Jie Cheng, Feng Liu, Feng Lin, Rendong Wu, Renji Zhang, Qingping Lu. 2006. Generation of Three-Dimensional Hepatocyte/Gelatin Structures with Rapid Prototyping System. Tissue Engineering, ahead of print060118075515002. [CrossRef]

19. Jyh-Ping Chen, Chia-Tseng Lin. 2006. Dynamic Seeding and Perfusion Culture of Hepatocytes with Galactosylated Vegetable Sponge in Packed-Bed Bioreactor. Journal of Bioscience and Bioengineering 102:1, 41-45. [CrossRef]

20. Xiaohong Wang, Yongnian Yan, Yuqiong Pan, Zhuo Xiong, Haixia Liu , Jie Cheng, Feng Liu , Feng Lin, Rendong Wu, Renji Zhang, Qingping Lu . 2006. Generation of Three-Dimensional Hepatocyte/Gelatin Structures with Rapid Prototyping SystemGeneration of Three-Dimensional Hepatocyte/Gelatin Structures with Rapid Prototyping System. Tissue Engineering 12:1, 83-90. [Abstract] [PDF] [PDF Plus]

21. Feng Zhao, Teng Ma. 2005. Perfusion bioreactor system for human mesenchymal stem cell tissue engineering: Dynamic cell seeding and construct development. Biotechnology and Bioengineering 91:4, 482-493. [CrossRef]

22. Joshua R. Mauney, Jeff Blumberg, Mono Pirun, Vladimir Volloch , Gordana Vunjak-Novakovic , David L. Kaplan . 2004. Osteogenic Differentiation of Human Bone Marrow Stromal Cells on Partially Demineralized Bone Scaffolds in VitroOsteogenic Differentiation of Human Bone Marrow Stromal Cells on Partially Demineralized Bone Scaffolds in Vitro. Tissue Engineering 10:1-2, 81-92. [Abstract] [PDF] [PDF Plus]

23. Yichao Wang, Toshimasa Uemura, Jian Dong, Hiroko Kojima , Junzo Tanaka, Tetsuya Tateishi . 2003. Application of Perfusion Culture System Improves in Vitro and in Vivo Osteogenesis of Bone Marrow-Derived Osteoblastic Cells in Porous Ceramic MaterialsApplication of Perfusion Culture System Improves in Vitro and in Vivo Osteogenesis of Bone Marrow-Derived Osteoblastic 
Cells in Porous Ceramic Materials. Tissue Engineering 9:6, 1205-1214. [Abstract] [PDF] [PDF Plus]

24. D. Wendt, A. Marsano, M. Jakob, M. Heberer, I. Martin. 2003. Oscillating perfusion of cell suspensions through three-dimensional scaffolds enhances cell seeding efficiency and uniformity. Biotechnology and Bioengineering 84:2, 205-214. [CrossRef]

25. Yoshitake Takahashi , Yasuhiko Tabata . 2003. Homogeneous Seeding of Mesenchymal Stem Cells into Nonwoven Fabric for Tissue EngineeringHomogeneous Seeding of Mesenchymal Stem Cells into Nonwoven Fabric for Tissue Engineering. Tissue Engineering 9:5, 931-938. [Abstract] [PDF] [PDF Plus]

26. Yascara Grisel Luna Saavedra, Mircea A. Mateescu, Diana A. Averill-Bates, Francine Denizeau. 2003. Polyvinylalcohol three-dimensional matrices for improved long-term dynamic culture of hepatocytes. Journal of Biomedical Materials Research 66A:3, 562-570. [CrossRef]

27. Mona Dvir-Ginzberg, Iris Gamlieli-Bonshtein, Riad Agbaria , Smadar Cohen . 2003. Liver Tissue Engineering within Alginate Scaffolds: Effects of Cell-Seeding Density on Hepatocyte Viability, Morphology, and FunctionLiver Tissue Engineering within Alginate Scaffolds: Effects of Cell-Seeding Density on Hepatocyte Viability, Morphology, and Function. Tissue Engineering 9:4, 757-766. [Abstract] [PDF] [PDF Plus]

28. Milica Radisic, Michelle Euloth, Liming Yang, Robert Langer, Lisa E. Freed, Gordana Vunjak-Novakovic. 2003. High-density seeding of myocyte cells for cardiac tissue engineering. Biotechnology and Bioengineering 82:4, 403-414. [CrossRef]

29. Juliette van den Dolder, Paul H.M. Spauwen , John A. Jansen . 2003. Evaluation of Various Seeding Techniques for Culturing Osteogenic Cells on Titanium Fiber MeshEvaluation of Various Seeding Techniques for Culturing Osteogenic Cells on Titanium Fiber Mesh. Tissue Engineering 9:2, 315-325. [Abstract] [PDF] [PDF Plus]

30. Juliette van den Dolder, Johan W. M. Vehof, Paul H. M. Spauwen, John A. Jansen. 2003. Bone formation by rat bone marrow cells cultured on titanium fiber mesh: Effect ofin vitro culture time. Journal of Biomedical Materials Research 62:3, 350-358. [CrossRef]

31. Jared W. Allen, Sangeeta N. Bhatia . 2002. Engineering Liver Therapies for the FutureEngineering Liver Therapies for the Future. Tissue Engineering 8:5, 725-737. [Abstract] [PDF] [PDF Plus]

32. Ivan MARTIN, Olivier DÉMARTEAU, Alessandra BRACCINI. 2002. Recent Advances in Cartilage Tissue Engineering: From the Choice of Cell Sources to the Use of Bioreactors. JSME International Journal Series C 45:4, 851-861. [CrossRef]

33. Tae Gwan Park. 2002. Perfusion culture of hepatocytes within galactose-derivatized biodegradable poly(lactide-co-glycolide) scaffolds prepared by gas foaming of effervescent salts. Journal of Biomedical Materials Research 59:1, 127-135. [CrossRef] 